\title{
Algorithm for optimization of power system short-term mode in conditions of partial uncertainty of initial information taking into account the frequency change
}

\author{
Tulkin Gayibov ${ }^{1, *}$ \\ ${ }^{1}$ Tashkent State Technical University named after Islam Karimov, Uzbekistan
}

\begin{abstract}
Many existing methods and algorithms for optimization of short-term modes of power systems provide in calculations the introduction of a slack bus station, which ensures the balance of active power and, accordingly, the permissible frequency. In cases where the real load deviates from the planned one, determined by forecasting, the power system mode may turn out to be not optimal, and sometimes even not acceptable. This factor is especially noticeable in conditions of partial uncertainty of initial information about loads of nodes. To overcome this problem, planning of power system mode should be carried out taking into account the frequency change and, accordingly, the regulatory capabilities of all stations.

This paper proposes an algorithm for optimization of modes of power systems in terms of partial uncertainty of initial information about loads, taking into account the frequency change. On the basis of computational experiments using the proposed algorithm, it is shown that taking into account the frequency change when planning modes of power systems with partially undefined loads of nodes can give a significant economic effect.
\end{abstract}

\section{Introduction}

Optimal planning of short-term modes of modern power systems refers to complex problems of nonlinear mathematical programming. It is characterized by the presence of a large number of variables of different scales, simple and functional constraints in the form of equalities and inequalities. Usually, such problems are solved on the basis of dividing the planned period (for example, a day) into small time intervals (for example, hours), within which the system is considered as a static system. To this period many different methods and algorithms have been created for solving of problems as a results of activities of many authors around the world. These include classical [1-3] and heuristic [4, 5] methods, as well as artificial computational intelligence methods based on ant algorithms [6,7], artificial neural networks $[8,9]$, cuckoo search [10], fuzzy logic [11], bees $[6,12,16]$, particle swarm optimization [13], evolution $[14,15]$, etc. Some of these methods and algorithms are already used to solve practical problems of production. At the same time, the need to take into account some additional factors, which take place for modern power systems require their corresponding improvement. One of the problems is associated with solving the problem with sufficient accuracy under conditions of partial uncertainty of used initial information about consumer loads and taking into account of frequency change. The existing methods for solving the problem under consideration are mainly adapted for using the deterministic initial information. Therefore, their application in conditions of partial uncertainty of initial information is possible on the basis of reducing the original problem to a number of successively solved deterministic problems by using the appropriate procedures as in [18-20]. On the other hand, calculation algorithms according to existing methods provide for the allocation of a single balancing station, which ensures the balance of active power and, accordingly, the allowable frequency in power system. However, in conditions of deviation of real loads of power systems from the values obtained by forecasting and the presence in power system several power plants participating in frequency regulation, the obtained results in basis of such algorithms can have significant errors that reduce the effect of optimization. Therefore, the development of algorithms for optimization of modes of power systems in conditions of partial uncertainty of initial information taking into account the frequency change is important problem. Such algorithms eliminate the need to select a single balancing power plant in the calculations and, accordingly, increase the optimization accuracy.

Below a new algorithm for power systems mode optimization under conditions of partial uncertainty of the initial information, taking into account the frequency change is proposed.

\footnotetext{
* Corresponding author: tulgayibov@gmail.com
} 


\section{The method of research}

In tasks of mode optimization of power system, information, mainly about active resistances and conductivities of network elements, as well as active loads of consumers, has a partially uncertain nature. Usually, the partially uncertain nature of the resistances and conductivities of elements depends on weather conditions and, mainly, on ambient temperature. Therefore, their partial uncertainty is taken into account by introducing appropriate corrections, taking into account the forecast of weather conditions for the planning period. But accounting of uncertainty of loads of consumers at nodes of power system is carried out in process of optimization of modes of power systems for each interval of short-term planning cycle.

As a result of optimal planning of short-term modes of power system, the optimal load schedules of power plants are determined. The implementation of optimal mode obtained for each time interval is carried out by power plants participating in optimization according to given dispatch schedules of loads. The deviation of active load of power system from the planned value leads to a deviation of frequency in power system. To maintain the frequency level within the permissible ranges, the total load deviation of the power system $\Delta P_{L}$ is covered by the power plants participating in frequency regulation, which usually have power reserve. In this case, the distribution of $\Delta P_{L}$ between such stations occurs in accordance with the static characteristics of their turbine speed controllers.

To illustrate the essence of described situation Fig. 1 shows the distribution of the total load $P_{L}$ and total deviation from it $\Delta P_{L}$ between the two units in accordance with the static characteristics of the turbine speed controllers.

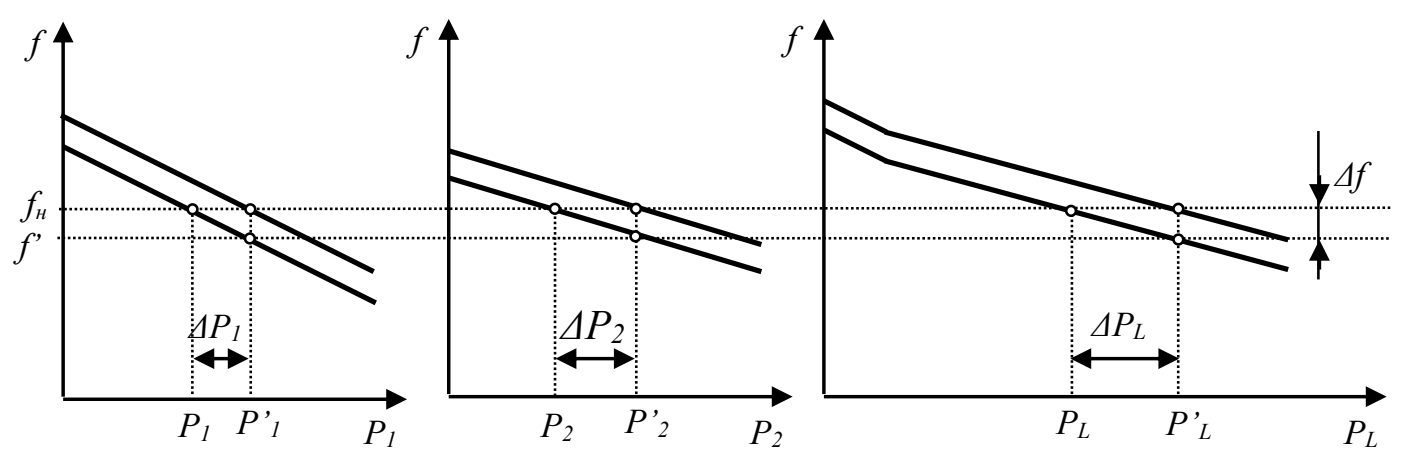

Fig. 1. Distribution of total load $P_{L}$ and deviation $\Delta P_{L}$ between units in accordance with the static characteristics of turbine speed controllers.

The total load $P_{L}$ is distributed between units at the rated frequency $f_{n}$ and their powers are equal to $P_{l}, P_{2}$, respectively. An increase in the load on $\Delta P_{L}$ is also distributed between them by similar way, as a result of their powers become

$$
P^{\prime}{ }_{1}=P_{1}+\Delta P_{1}, \quad P_{2}{ }_{2}=P_{2}+\Delta P_{2} \text {. }
$$

This will set the frequency $f^{\prime}$ in power system. If the frequency $f^{\prime}$ is less than the minimum permissible value, then the turbine speed controllers operate in units, under the influence of which the static characteristics of units rise by $\Delta f$ relative to the initial positions. The distribution of total load $P_{L}+\Delta P_{L}$ takes place according to the new obtained static characteristics and the nominal frequency is restored with the new powers of the units.

The coverage of deviations of total load in power system carried out by power plants participating in frequency control in accordance with the static characteristics of turbines of units in them.

The use of graphical method to determine of new powers of stations causes certain inconveniences and inaccuracies in calculations. Therefore, it is advisable to use the following analytical expressions for this purpose:

$$
\begin{gathered}
\Delta P_{1}=k_{1} \Delta f, \quad P_{2}=k_{2} \Delta f, \\
\Delta P_{1}+\Delta P_{2}=\Delta P_{H}, \\
\left(k_{1}+k_{2}\right) * \Delta f=\Delta P_{H}, \\
\Delta f=\Delta P_{H} /\left(k_{1}+k_{2}\right),
\end{gathered}
$$

where $k_{1}, k_{2}$ are static coefficients of turbine speed controllers at power stations.

\subsection{The algorithm of optimization}

To explain the essence of proposed algorithm for optimization of mode under conditions of partial uncertainty of initial information taking into account the frequency change, let us consider the power system with four thermal power plants (TPP) participating in optimization.

The optimization problem for each interval of the planning period is formed as follows:

minimize the objective function, consisting of function of total fuel costs or the equivalent fuel consumption in all $n$ TPPs

$$
B=\sum_{i=1}^{n} B_{i}\left(P_{i}\right)
$$

subject to constraints on of power balance in power system

$$
\sum_{i=1}^{n} P_{i}=P_{u}
$$

and maximum and minimum possible power plant capacities

$$
P_{i}^{\min } \leq P_{i} \leq P_{i}^{\max }, i=1,2, \ldots, n .
$$

The total load of power system $P_{L}$ is partially uncertain, i.e. only the limiting maximum and minimum 
values are known. Moreover, the loads inside the segment are not assigned any probabilistic characteristics, since they are unknown.

Let us describe the proposed algorithm for optimization of modes of power systems under conditions of partial uncertainty of initial information taking into account the frequency change:

1) In given range of possible load values, a set of values $\left\{P_{L 1}, P_{L 2}, \ldots, P_{L n}\right\}$ is selected and $P_{L 1}=P_{\mathrm{L}}^{m i n}$ and $P_{L n}=P_{L}^{\max }$. In this case, the number of possible load values in a given range is recommended to be selected with the condition of ensuring the required accuracy of solution of the problem and an acceptable amount of computational operations performed.

The total loads of power system $P_{L 1}, P_{L 2}, \ldots, P_{L n}$ are optimally distributed between the stations, i.e. problem (5) - (7) is solved for different values of total load. For this purposes it is possible to use the existing optimization methods in conditions of deterministic initial information, and in cases of discontinuity of power characteristics of stations, the algorithm given in [18].

The optimal solutions obtained at this stage forms conditionally optimal plans for the problem. Then, at these conditionally optimal plans and corresponding loads of electric consumers the values of objective function which form the diagonal elements of the "payment matrix" $B_{i i}, i=1,2, \ldots, n$ are calculated as in optimization under conditions of probabilistic nature of initial information [17].

2) The values of objective function for each of conditionally optimal plans under all possible conditions for their implementation, i.e. for total loads of power system, are calculated. At this stage, the deviation in total load, obtained due to the difference of total loads used in obtaining the corresponding conditionally optimal plan and other possible load is distributed between the stations involved in frequency control in accordance with static characteristics of their turbine speed controllers. In this case, the frequency deviation is determined by formula (3), and the corresponding powers of stations - by (1). The obtained values of objective function are used to form offdiagonal elements of "payoff matrix" $B_{i j}, i=1,2, \ldots, n$; $j=1,2, \ldots, n(i \# j)$.

3) The choice of the best plan among conditionally optimal plans in basis of the use of received payment matrix is carried out by additional criterion of minimax risk, which proceeds from the assumption that no matter what plan we accept, the risk from its implementation will necessarily be the worst $[17,21$, 22 ].

In this case, it is reasonable to accept as optimal the conditionally optimal plan for which the maximum value of the objective function risk is less than its maximum value for any other conditionally optimal plan. This objective function value can be described as

$$
R_{\text {on }}=\min (k) \max (j) R_{k j},
$$

where $R_{k j}=B_{k j}-B_{j j}$.

\section{Results and discussion}

The efficiency of the given algorithm is investigated by the example of optimal distribution of total partially uncertain load of power system between four TPPs taking into account the frequency change. The characteristics of equivalent fuel consumption of TPPs are given below (in tones of fuel equivalent or t.f.e. in short).

$$
\begin{gathered}
B_{1}=100+0.2 * P_{1}+0.002 * P_{1}^{2} ; \\
B_{3}=60+0.15 * P_{3}+0.0015 * P_{3}^{2} ; \\
B_{2}=120+0.2 * P_{2}+0.0025 * P_{2}^{2} ; \\
B_{4}=80+0.25 * P_{4}+0.001 * P_{4}^{2} .
\end{gathered}
$$

The total load of power system is partially uncertain [1620 MW, $1780 \mathrm{MW}]$.

Power plants TPP-1 and TPP-3 participating in frequency regulation in power system. The static coefficients of turbine speed controllers in these stations are $k_{1}=0.2$ and $k_{3}=0.15$, respectively.

To optimize according to the proposed algorithm the given range of total load of power system is divided into five intervals with the capacities given in Table 1.

Table 1. Total loads of power system.

\begin{tabular}{|c|c|c|c|c|c|}
\hline $\begin{array}{c}\text { Interval } \\
\text { number }\end{array}$ & 1 & 2 & 3 & 4 & 5 \\
\hline$P_{L}, M W$ & 1620 & 1660 & 1700 & 1740 & 1780 \\
\hline
\end{tabular}

Optimization of the power system mode taking into account the frequency change by the algorithm described above is carried out. Table 2 shows the obtained conditionally optimal plans.

Table 2. Conditionally optimal plans obtained as a result of optimal distribution of total loads of power system between TPPs.

\begin{tabular}{|c|c|c|c|c|c|}
\hline \multirow{2}{*}{$\begin{array}{c}\text { Plan } \\
\text { No }\end{array}$} & \multirow{2}{*}{$\begin{array}{c}\text { Total } \\
\text { load, } \\
\text { MW }\end{array}$} & \multicolumn{4}{|c|}{ Conditional optimal powers of TPP, MW } \\
\cline { 3 - 6 } & $P_{1}$ & $P_{2}$ & $P_{3}$ & $P_{4}$ \\
\hline 1 & 1485 & 290,91 & 232,73 & 404,54 & 556,82 \\
\hline 2 & 1567.5 & 306,98 & 245,58 & 425,97 & 588,96 \\
\hline 3 & 1650 & 323,05 & 258,44 & 447,40 & 621,10 \\
\hline 4 & 1732.5 & 339,12 & 271,30 & 468,83 & 653,25 \\
\hline 5 & 1815 & 355,19 & 284,15 & 490,26 & 685,39 \\
\hline
\end{tabular}

The choice from these conditionally optimal plans the optimal one was carried out on the basis of using the received payment matrix (shown in Table 3) with the elements calculated at powers of stations shown in Table 2 and the corresponding conditions for their implementation at various possible total loads of power system. 
Table 3. Payment matrix at participation in frequency regulation of two TPPs.

\begin{tabular}{|c|c|c|c|c|c|}
\hline \multirow{2}{*}{$\begin{array}{c}\text { Plan } \\
\text { No }\end{array}$} & \multicolumn{5}{|c|}{ The total load of power system, MW } \\
\cline { 2 - 6 } & 1485 & 1567,5 & 1650 & 1732,5 & 1815 \\
\hline 1 & 1524,81 & 1643,14 & 1773,14 & 1914,81 & 2068,15 \\
\hline 2 & 1527,97 & 1639,94 & 1763,58 & 1898,89 & 2045,86 \\
\hline 3 & 1537,52 & 1643,13 & 1760,40 & 1889,34 & 2029,95 \\
\hline 4 & 1553,45 & 1652,69 & 1763,60 & 1886,17 & 2020,42 \\
\hline 5 & 1575,74 & 1668,58 & 1773,13 & 1889,31 & 2017,22 \\
\hline
\end{tabular}

According to the criterion of minimax risk, the third conditionally optimal plan was obtained as the optimal one, in which the highest possible total fuel consumption is 2029.95 t.f.e. / h.

To compare and evaluate the effectiveness of the proposed optimization algorithm taking into account the frequency change under conditions of partial uncertainty of initial information about the loads of power system the problem under consideration was also solved by the traditional algorithm, i.e. with the allocation of the single slack bus station of TPP-1. The payment matrix for this case is presented in Table 4.

Table 4. Payment matrix at optimization with the allocation of single slack bus station TPP-1.

\begin{tabular}{|c|c|c|c|c|c|}
\hline \multirow{2}{*}{$\begin{array}{c}\text { Plan } \\
\text { No }\end{array}$} & \multicolumn{5}{|c|}{ The total load of power system, MW } \\
\cline { 2 - 6 } & 1485 & 1567,5 & 1650 & 1732,5 & 1815 \\
\hline 1 & 1524,81 & 1650,92 & 1804,26 & 1984,82 & 2192,61 \\
\hline 2 & 1535,76 & 1639,96 & 1771,38 & 1930,02 & 2115,89 \\
\hline 3 & 1568,64 & 1650,92 & 1760,41 & 1897,14 & 2061,08 \\
\hline 4 & 1623,46 & 1683,80 & 1771,37 & 1886,17 & 2028,19 \\
\hline 5 & 1700,17 & 1738,60 & 1804,25 & 1897,13 & 2017,23 \\
\hline
\end{tabular}

The choice of the optimal plan according to the obtained payment matrix based on the use of minimax risk criterion gives the 3 rd conditionally optimal plan, in which the maximum possible total fuel consumption is 2061.08 t.f.e / h.

Comparing the results of optimization of power system mode under conditions of partial uncertainty of initial information about loads taking into account the frequency change by the proposed algorithm, when two TPPs are involved in covering the power imbalances, with the results of optimization by selecting a single balancing slack bus station, we will make sure that taking into account the frequency change in solving the problem can give significant economic effect. In the example considered, the hourly savings in the equivalent fuel consumption due to optimization under conditions of partial uncertainty of initial load taking into account of frequency change by the proposed algorithm was 31.13 t.f.e./h. or $1.5 \%$ of total consumption of equivalent fuel in all four TPPs.

\section{Conclusion}

1. An algorithm for optimization of modes of power systems under conditions of partial uncertainty of initial information taking into account the frequency change, which eliminates the need to select a single balancing station in the calculations is proposed.

2. It was revealed that taking into account the frequency change in optimization of modes of power systems under conditions of partial uncertainty of initial information about the loads can provide a significant economic effect by reducing the total fuel consumption in thermal power plants.

3. The proposed optimization algorithm under conditions of partial uncertainty of initial information taking into account the frequency change can be used by dispatching services of power systems for optimal planning of short-term modes and operational control of power system with several stations participating in frequency regulation.

\section{References}

1. J. Zhu, Optimization of Power System Operation (Wiley-IEEE Press, 2015).

2. M.S. Bazaraa, H.D. Sherali, C.M. Shetty, Nonlinear Programming: Theory and Algorithms (Wiley, 2006).

3. M.E. El-Havary, G.S. Christenstn, Optimal Economic Operation of Electric Power Systems (Academic Press, New York, 1979).

4. X.S. Yang, Nature-inspired metaheuristic algorithms (Luniver Press, 2010).

5. Z. Michalewicz, D.B. Fogel, How to Solve It. Modern Heuristics (Springer, 2004).

6. A. Colorni, M. Dorigo, V. Maniezzo, ECAL91 (Elsevier Publishing, Paris, 1991).

7. Linda Slimani, Tarek Bouktir, International Review of Electrical Engineering, 6(7) (2011).

8. K. Abaci, V. Yamacli, Advances in Electrical and Computer Engineering, 19(4) (2019).

doi:10.4316/AECE.2019.04007.

9. P.J. Braspenning, F. Thuijsman, A.J.M.M. Weijter, Artificial Neural Networks: An Introduction to ANN Theory and Practice (Springer, 1995).

10. X.S. Yang, S. Deb, 2009 World Congress on Nature \& Biologically Inspired Computing (IEEE, Coimbatore, 2009). doi:10.1109/NABIC.2009.5393690.

11. W. Pedrycz, Fuzzy control and fuzzy systems (John Wiley \& Sons, 1996).

12. D.T. Pham, A. Ghanbarzadeh, E. Koc, Intelligent Production Machines and Systems (Cardiff, UK, Elsevier, 2006). doi: 10.1016/B978-0080451572/50081-X.

13. P. Pijarski, P. Kacejko, Advances in Electrical and Computer Engineering. 18(4) (2018). 
doi:10.4316/AECE.2018.04005.

14 J. Yuryevich K.P. Wong, Evolutionary Programming Based Optimal Power Flow Algorithm, 14(4) (IEEE Transaction on power Systems, 1999).

15. J.S.Al-Sumait, J.K. Sykulski A.K. AlOthman, Electric Power Components and Systems 36(3) (2008). doi:10.1080/15325000701603892.

16. B. Allaoua, A. Laoufi, Advances in Electrical and Computer Engineering 9(1) (2009). doi:10.4316/AECE.2009.01006.

17. T Gayibov, Sh Latipov, D Abdurashidov, B Pulatov, A Davirov. IOP Conf. Series: Materials Science and Engineering, 883, 012185 (2020). doi:10.1088/1757-899X/883/1/012185.

18. Tulkin Gayibov, Sherxon Latipov, Bakhadir Uzakov. Rudenko International Conference "Methodological problems in reliability study of large energy systems" E3S Web of Conference, 139, 01086 (2019). https://doi.org/10.1051/e3sconf/201913901086.

19. M. Valdma, M. Keel, O. Liik, H. Tammoja, Proccedings of IEEE Bologna PowerTech (2003).

20. M. Valdma, M. Keel, O. Liik, Proccedings. of Tenth Power Systems Computation Conference, (Graz, Austria 1990).

21. T.Sh. Gayibov, Sh.Sh. Latipov, Technical science and innovation, 2(2) (2019).

22. Tulkin Gayibov, Bekzod Pulatov, Sherxon Latipov, Gulnaz Turmanova, Rudenko International Conference "Methodological problems in reliability study of large energy systems" E3S Web of Conference, 139, 01031 (2019).

https://doi.org/10.1051/e3sconf/201913901031 